\title{
Abdominal Wall Reconstruction with a Free Fibula Flap after Internal Extended Hemipelvectomy
}

\author{
Pedro C. Cavadas ${ }^{1}$ Daniela Téllez-Palacios² \\ ${ }^{1}$ Department of Reconstructive Surgery, Clínica Cavadas, \\ Valencia, Spain \\ 2Department of Reconstructive Microsurgery, Clínica Cavadas, \\ Valencia, Spain

\author{
Address for correspondence Pedro C. Cavadas, MD, PhD, \\ Department of Reconstructive Surgery, Clínica Cavadas, Paseo de las \\ Facultades 1 bajo 8, Valencia, 46021, Spain \\ (e-mail: pcavadas@clinicacavadas.es).
}

\begin{abstract}
Keywords

- osteosarcoma

- hemipelvectomy

- fibula flap

Major pelvic resections for malignant tumors are infrequent and have significant morbidity and mortality, for instance, incisional hernias are postoperative complications uncommonly reported probably because most cases are overshadowed by more serious complications. Reconstruction depends on the extent of the resection and overall prognosis of the patient. A case of a late complex hypogastric and femoral incisional hernia after extended hemipelvectomy for recurrent osteosarcoma treated with distal abdominal wall fixation into a free fibula flap is reported.
\end{abstract}

\section{Introduction}

Major pelvic resections for malignant tumors of the pelvic ring are infrequent, extensive surgical procedures with relatively high incidence of postoperative complications and significant mortality. ${ }^{1}$ Hemipelvectomies are classified as internal or external depending on the preservation or amputation of the lower limb, respectively. Complication rates are high in both types. ${ }^{2,3}$ The case of internal hemipelvectomies reconstruction depends on the extent of the resection and overall prognosis of the patient, with a remarkable lack of consensus about the need of bone reconstruction and the optimal reconstructive technique if performed.

Incisional hernia is an uncommonly reported postoperative complication of hemipelvectomies, and most reported cases refer to external hemipelvectomies in the form of hernia of the pelvic stump. ${ }^{4-7}$ Few cases of incisional hernia have been reported after internal hemipelvectomy probably because most cases are asymptomatic or overshadowed by more serious complications. ${ }^{8}$

A case of late complex hypogastric and femoral incisional hernia after extended hemipelvectomy for recurrent osteosarcoma treated with distal abdominal wall fixation into a free fibula flap is reported. To the best of the authors' knowledge, no similar technique has been described previously in the English literature.

\section{Case Report}

A 32-year-old male patient presented with a recurrent osteosarcoma of the left innominate bone after two previous attempts at local resection. The tumor was $13 \mathrm{~cm}$ in diameter, affecting the left iliac and pubic bones, acetabulum, adductor compartment through the obturator canal, left ureter, urinary bladder, left spermatic cord, and prostate (-Fig. 1). The tumor was resected including all affected structures through an extended internal hemipelvectomy. The left femoral vein was resected and bypassed with a greater saphenous graft. The femoral artery and the femoral nerve were preserved. Prostate, partial bladder, and partial sigma resection were performed, and a left-to- right transureter ureterostomy reestablished the left urinary way. The left testes and cavernous corpus were also resected. The left lower abdominal wall was reinforced with a polypropylene mesh and a femoroiliac arthrodesis was performed with $4.5-\mathrm{mm}$ reconstruction plates. Histological clear margins were obtained. During the early postoperative period, three abdominal revisions due to recurrent uroperitoneum were needed with left cutaneous ureterostomy.

One year postoperative, a bladder augmentation procedure was performed with an ileocystoplasty with left ureter reinsertion, without complications.

Six years after the index surgery, with no evidence of tumor progression, the patient complained of a large hypogastric

(C)2020 Association of Plastic Surgeons of India
License terms

()(1) $\Theta \circledast$ published online

April 1, 2020
DOI https://doi.org/

$10.1055 / \mathrm{s}-0040-1709378$

ISSN 0970-0358. 
incisional hernia with abdominal contents herniating to the adductor and femoral regions. The lack of bone in the left pelvic ring precluded anchoring the left lower abdominal wall into a solid structure ( - Fig. 2).

A free fibula flap was harvested from the right side and used, with one osteotomy, to reconstruct the iliopubic continuity, caudal to the femoral vessels and femoral nerve. Bone fixation was performed with $3.5 \mathrm{~mm}$ locking reconstruction plates and the flap pedicle was anastomosed to the right

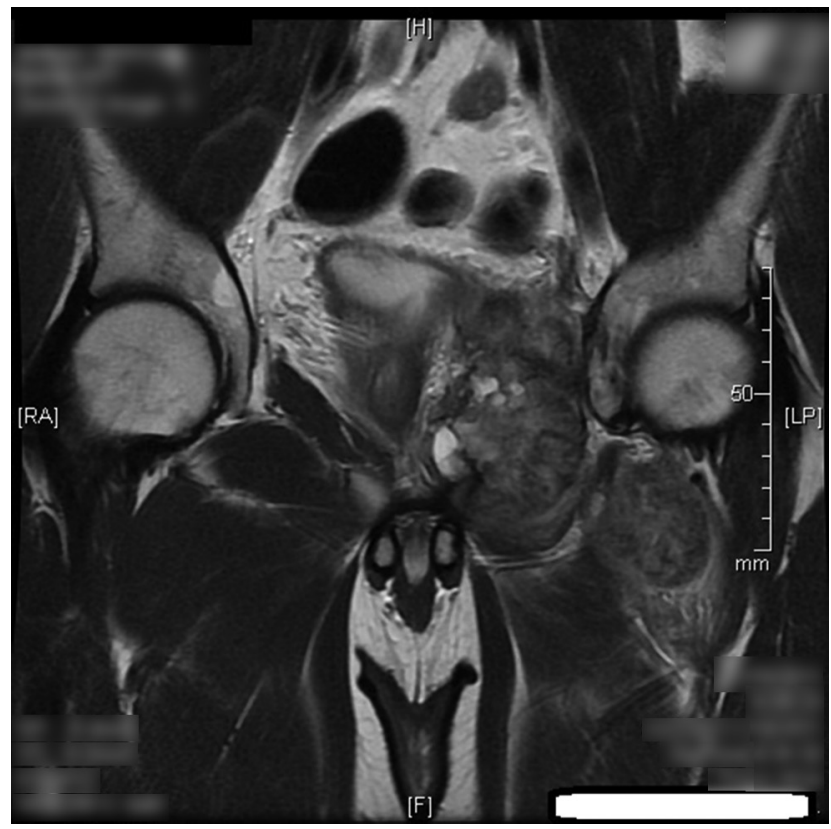

Fig. 1 Extent of the recurrent osteosarcoma of the left hemipelvis, with infiltration of the adductor compartment, prostate, urine bladder, and sigma. femoral artery and greater saphenous vein through a single-stage vascular loop (-Figs. 3 and 4). Four months later, once the bone consolidation was solid enough, the lower abdominal wall was reinserted using multiple bone suture anchors to the fibula (-Fig. 5). The postoperative course was uneventful with correction of the hernia. At the time, the patient is alive, active, in complete oncological remission and with a competent abdominal wall (-Video 1). Erectile dysfunction and fixed hip extension are the only complaints of the patient.

\section{Discussion}

Internal hemipelvectomy for malignant tumor resection is a major surgical procedure with potential severe complications and nonnegligible mortality. Osseous reconstruction using double barreled free fibula flaps has been reported with good long-term results, although the need for osseous reconstruction of the pelvic rim after internal hemipelvectomy for load transmission is still a subject of debate. ${ }^{9,10}$

Abdominal wall complications are rarely reported after internal hemipelvectomy. ${ }^{8}$ In standard anterior hemipelvectomies, there is enough muscular mass in the adductor compartment to allow for a solid repair of the lower abdominal wall and inferior (adductor or femoral) hernias are uncommon. In the case presented herein, the extent of resection included the adductor compartment resulting in a weaker-than-desired repair of the pelvic floor, despite the use of polypropylene mesh. The lack of a solid structure to suture the incompetent inferior abdominal wall raised the need for delayed bone reconstruction of the anterolateral pelvis. Load bearing through the left iliofemoral arthrodesis allowed the patient asymptomatic daily walking without

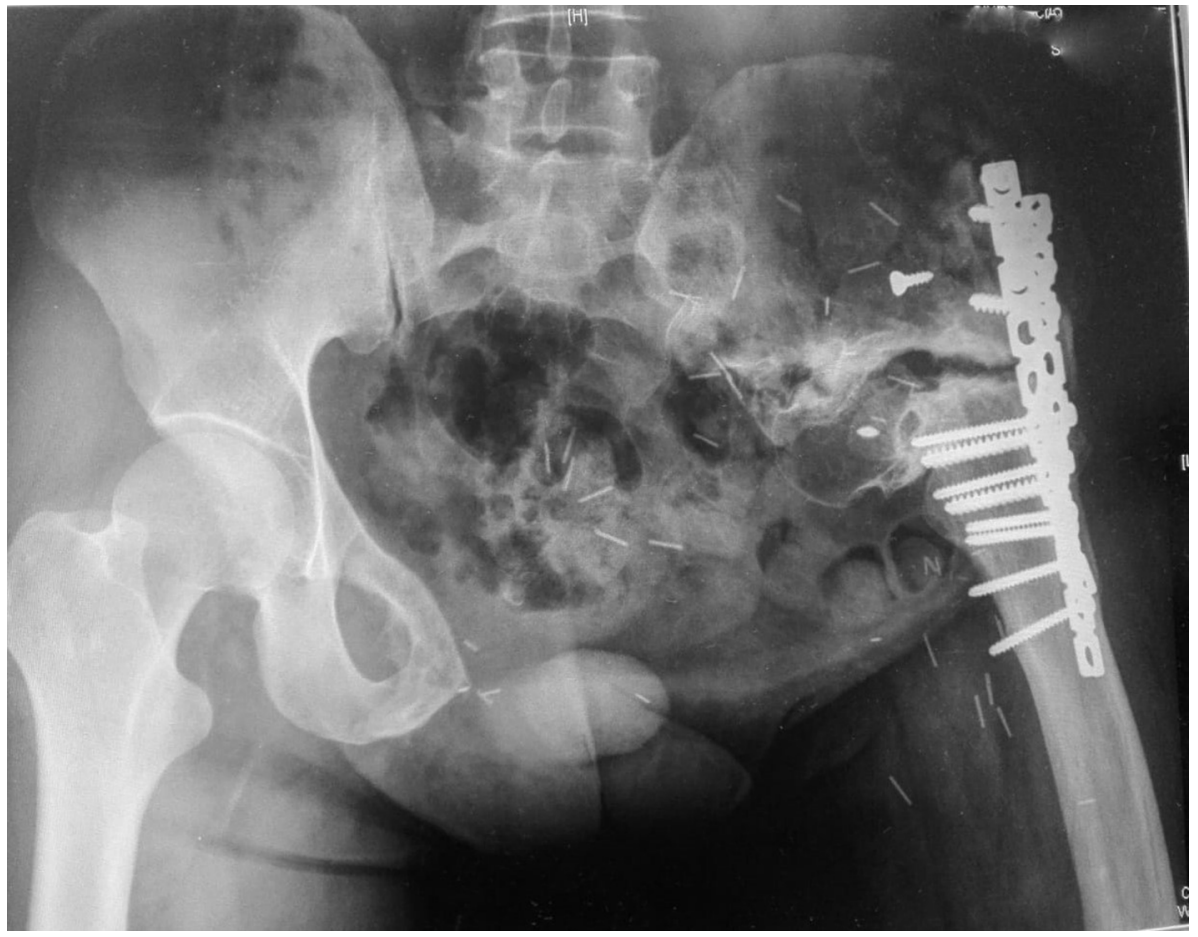

Fig. 2 Postoperative X-ray with iliofemoral arthrodesis. No load-bearing issues in the daily walking. 


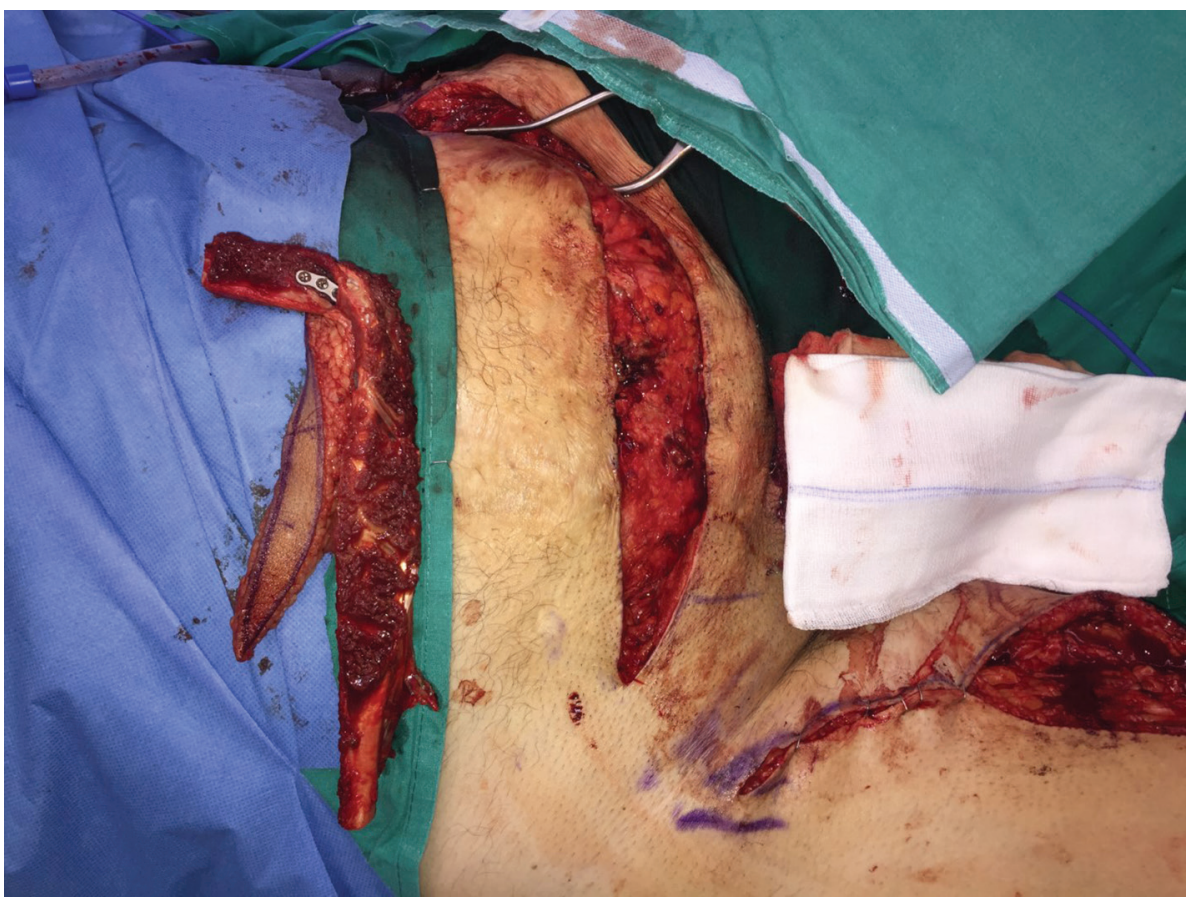

Fig. 3 A right free fibula osteocutaneous flap was used to reconstruct the pelvic rim. Anastomoses were performed to the right femoral vessels through a vascular loop with the greater saphenous vein. Locking $3.5-\mathrm{mm}$ reconstruction plates were used for fixation.

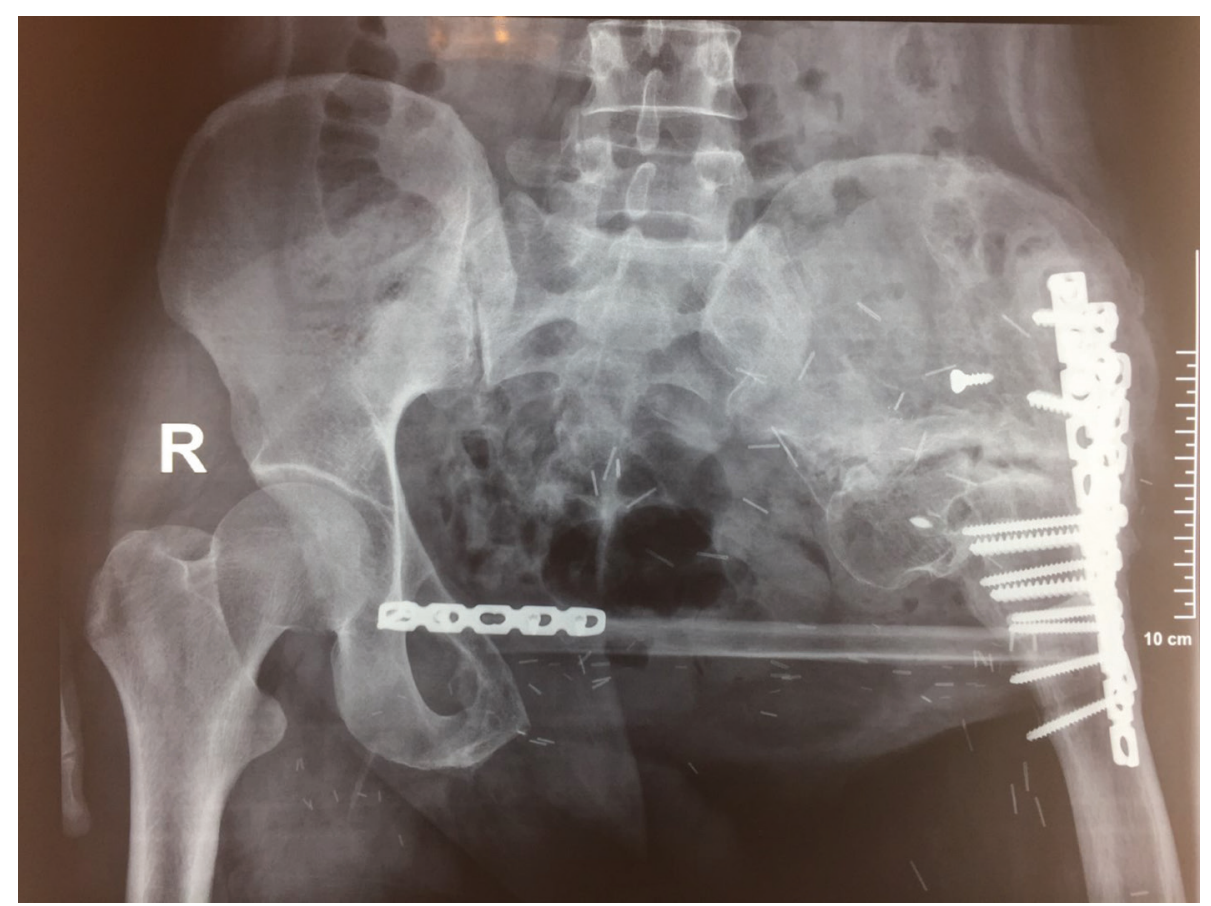

Fig. 4 X-ray of the bone reconstruction, prior to abdominal wall anchoring.

further bone reconstruction. The indication for skeletal pelvic ring reconstruction in this case was not for load transmission but to provide a solid structure for abdominal wall anchoring. The contralateral fibula flap was ideal in terms of length, cross-section, and healing potential in an irradiated area. Staging the reconstruction allowing the bone to heal before multiple drill holes and bone anchors were placed and a high-tensioned abdominal wall repair was performed seemed logical from biological and mechanical standpoints.

\section{Conclusion}

The reconstruction could have been performed earlier, at the time of the hemipelvectomy, but the amount of surgical 


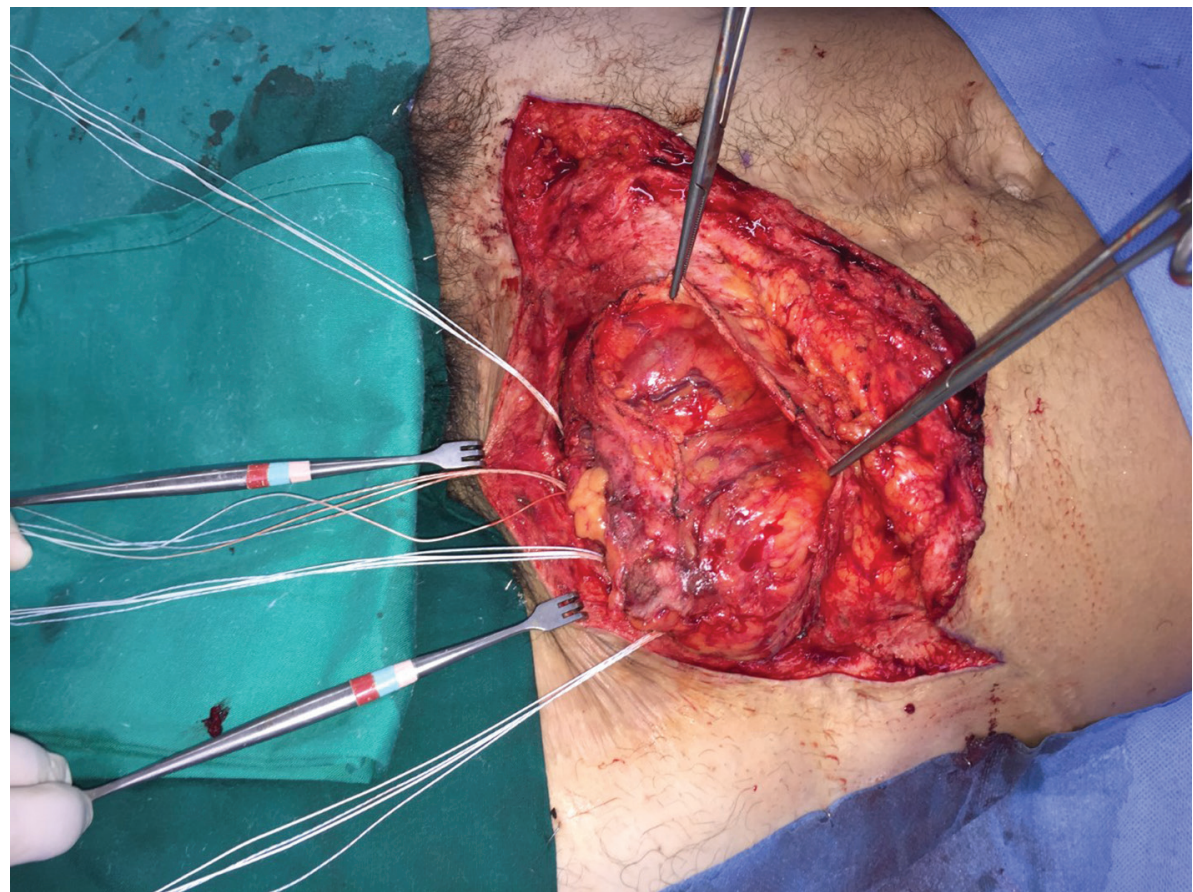

Fig. 5 Multiple heavy polyester sutures were fixed to the consolidated free fibula flap, respecting the passage of the femoral nerve and the femoral vessels.

\section{Video 1}

The patient performing Valsalva maneuvers, showing a competent abdominal wall. Online content including video sequences viewable at: www.thieme-connect.com/ products/ ejournals/html/10.1055/s-0040-1709378.

insult in an extended internal hemipelvectomy is massive and adding a free flap increases morbidity and potential postoperative systemic complications. The possibility of a massive adductor and femoral abdominal incisional hernia was not foreseen, due to the rarity of this complication. Multiple abdominal revisions in the early postoperative period may have contributed to the poor quality of the abdominal wall healing.

\section{Conflict of Interest}

None declared.

\section{References}

1 Bickels J, Malawer M. Pelvic resections (internal hemipelvectomies). In: Malawer MM, Sugarbaker PH, eds. Musculoskeletal Cancer Surgery. Treatment of Sarcomas and Allied Diseases. New York, NY: Kluwer Academic Publishers; 2001 405-414
2 Guder WK, Hardes J, Gosheger G, Henrichs MP, Nottrott M, Streitbürger A. Analysis of surgical and oncological outcome in internal and external hemipelvectomy in 34 patients above the age of 65 years at a mean follow-up of 56 months. BMC Musculoskelet Disord 2015;18:16-33

3 Couto AG, Araújo B, Torres de Vasconcelos RA, Renni MJ, Da Fonseca CO, Cavalcanti IL. Survival rate and perioperative data of patients who have undergone hemipelvectomy: a retrospective case series. World J Surg Oncol 2016;14(1):255

4 Bhattacharya S, Biswas S. Post hemipelvectomy incisional hernia. Hernia 2009;13(4):435-437

5 Kraybill WG, Standiford SB, Johnson FE. Posthemipelvectomy hernia. J Surg Oncol 1992;51(1):38-41

6 Apffelstaedt JP, Driscoll DL, Spellman JE, Velez AF, Gibbs JF, Karakousis CP. Complications and outcome of external hemipelvectomy in the management of pelvic tumors. Ann Surg Oncol 1996;3(3):304-309

7 Holbert JM, Lewis E. CT evaluation of the pelvis after hemipelvectomy. AJR Am J Roentgenol 1985;145(6):1233-1239

8 Arkoulis N, Savanis G, Simatos G, Zerbinis H, Nisiotis A. Incisional hernia of the urinary bladder following internal hemipelvectomy. Int J Surg Case Rep 2012;3(7):316-318

9 Ogura K, Sakuraba M, Miyamoto S, Fujiwara T, Chuman H, Kawai A. Pelvic ring reconstruction with a double-barreled free vascularized fibula graft after resection of malignant pelvic bone tumor. Arch Orthop Trauma Surg 2015;135(5):619-625

10 Sakuraba M, Kimata Y, Iida H, Beppu Y, Chuman $H$, Kawai A. Pelvic ring reconstruction with the double-barreled vascularized fibular free flap. Plast Reconstr Surg 2005;116(5):1340-1345 